\title{
Single-implant mandibular overdenture: a cost-effectiveness analysis
}

Leles CR, Nogueira TE, Resende GP, Bandeira ACM, Hartmann R, Aguiar FMO, Rios LF

Federal University of Goias, Goiania, Brazil

\section{Background}

Evidences from clinical studies show that the simplified mandibular overdenture (OVD) using a single implant:

- improve patient outcomes compared to CD [Nogueira et al., 2017];

- is similar to the 2-implant OVD [Bryant et al., 2015];

- has high implant survival rates [Srinivasan et al., 2016];

- can be a reliable alternative for older patients [Passia et al, 2015];

- is a simpler, less invasive and less costly option [Walton et al., 2009].

\section{Glinical question / hypothesis}

Is it worth to shift from a complete denture (CD) to a single implant mandibular overdenture?

We hypothesized that: (1) this is a safe and reliable intervention and (2) treatment results can be highly improved with relatively low costs.

\section{Aim}

To compare costs and consequences of treating conventional completed denture wearers with a single-implant mandibular OVD.

\section{Methods}

- Design: parallel two-arms clinical trial alongside a CEA

- Interventions: CD (control) versus single implant overdenture

- Participants: 123 screened $\rightarrow 39$ excluded $\rightarrow 84$ randomized

- Mean age 65.4 ( \pm 8.9 ); 58 female; No between-groups differences

\section{Study flowehart:}

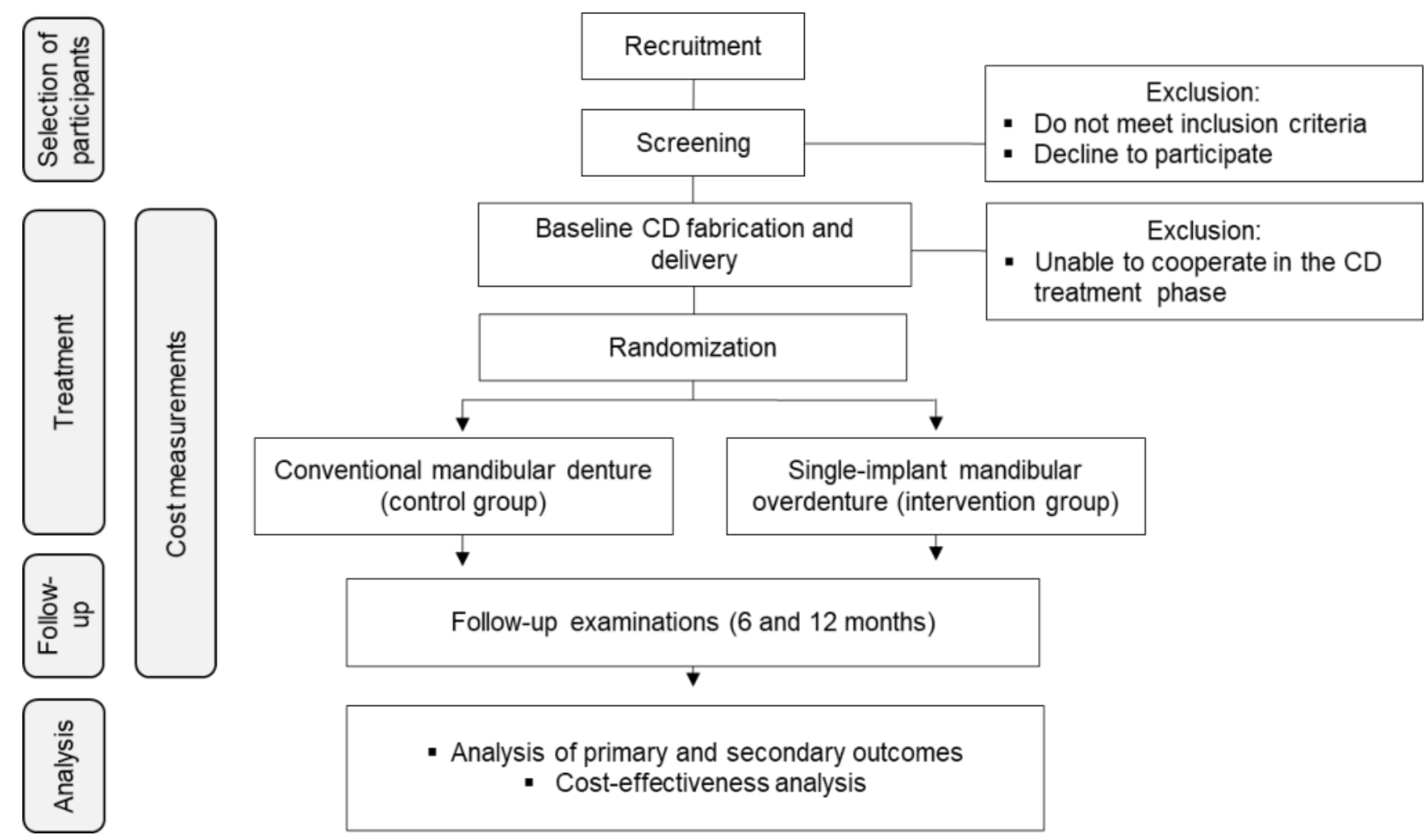

\section{Implant procedures (surgery and prosthesis):}

- 3.75mm diameter, external hexagon implant and O'ring-ball attachment (Neodent, Curitiba, Brazil);

- Immediate loading $\rightarrow$ insertion torque $>30$ and ISQ $>60$.
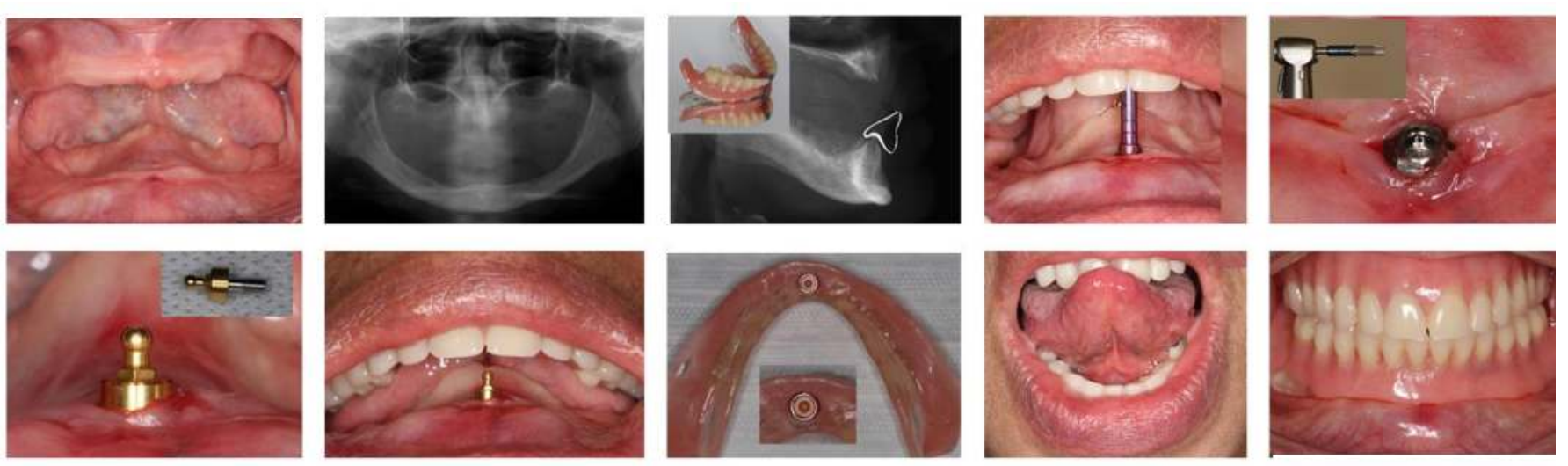

Outcomes:

- Satisfaction with the dentures (6-items 0-100 ordinal scale);

- Oral health-related quality of life: OHIP-Edent (Brazilian version).

\section{Economic analysis:}

- Perspective of the Brazilian public health system;

- Relevant direct costs: manpower, consumables, laboratory, implant/component costs ("bottom-up" costing estimation);

- $\mathrm{BRL} \$ \rightarrow 2016$ PPP conversion rate (1 PPP US\$=1.995 BRL);

- Incremental cost-effectiveness ratio (ICER);

- Modeling: decision tree (roll-back calculation method).

\section{Results}

OVD group: after 12-mo $(\mathrm{n}=31)$ :

- 9 refused implant insertion

- 1 implant failure (97\% survival)

- 1 withdrawal

- 32/33 immediately loaded

\section{Effectiveness and costs}
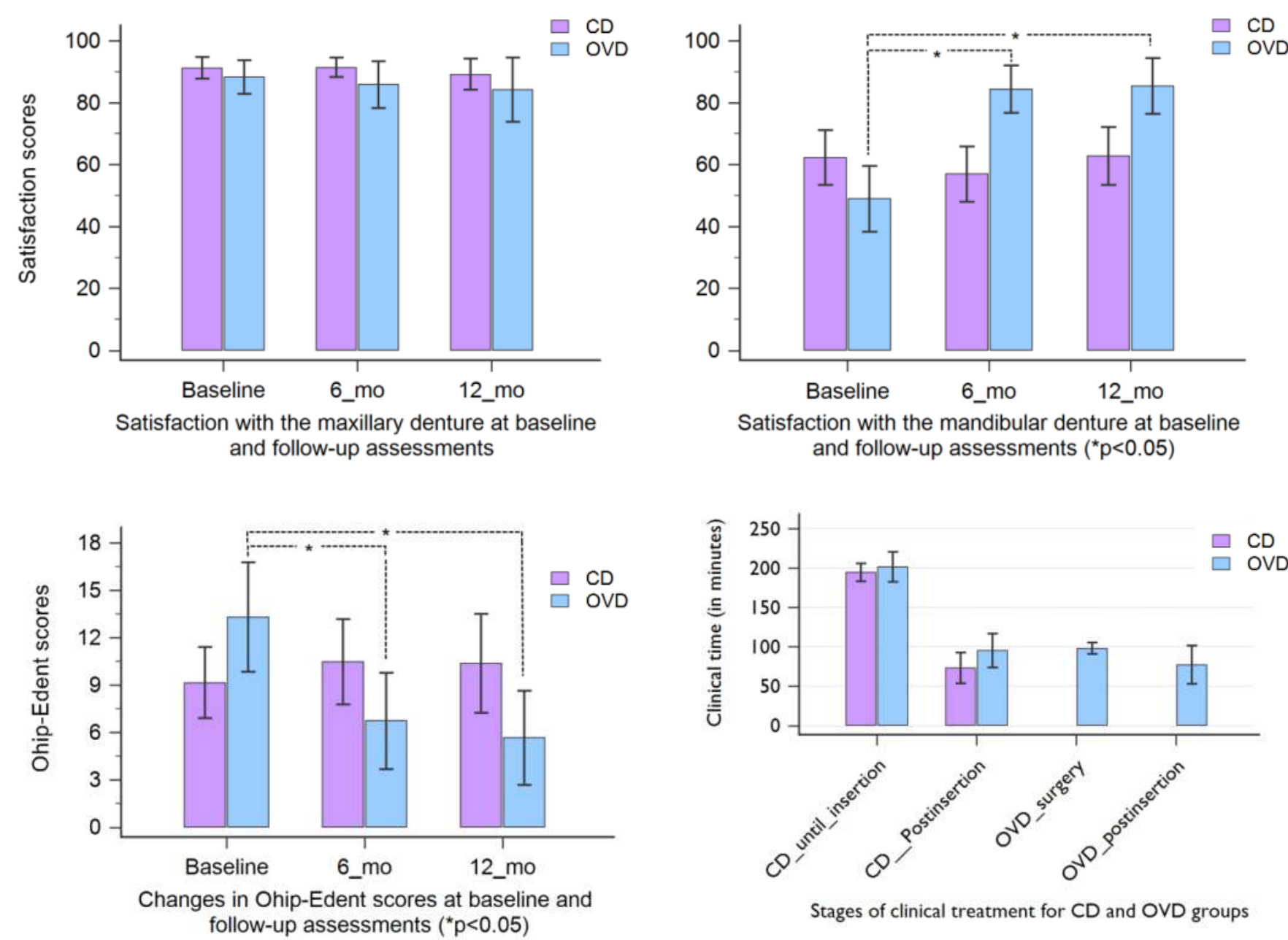

\begin{tabular}{lcccc}
\multirow{2}{*}{ Total treatment direct costs converted to Us\$ according to PPP } \\
\cline { 2 - 5 } Items & \multicolumn{3}{c}{ BRL \$ } & PPP Converted \\
\hline Consumables & 207.54 & 273.17 & 104.03 & 136.93 \\
Laboratory & 393.45 & 410.11 & 197.22 & 205.57 \\
Implants and components & - & 358.30 & - & 179.60 \\
Exams & - & 40.67 & - & 20.39 \\
Medication & - & 14.67 & - & 7.35 \\
Clinical time & 107.00 & 185.78 & 53.63 & 93.12 \\
\hline \multirow{2}{*}{ Total } & $\mathbf{7 0 7 . 9 9}$ & $\mathbf{1 2 8 2 . 7 0}$ & $\mathbf{3 5 4 . 8 8}$ & $\mathbf{6 4 2 . 9 6}$ \\
\hline
\end{tabular}

\begin{tabular}{|c|c|c|c|c|c|c|c|c|}
\hline \multicolumn{9}{|c|}{ Cost-effectiveness analysis } \\
\hline \multirow[b]{2}{*}{ Outcome } & \multirow[b]{2}{*}{ Group } & \multicolumn{2}{|c|}{ Outcome measure } & \multirow[b]{2}{*}{ Effectiveness } & \multirow[b]{2}{*}{$\begin{array}{l}\text { Incremental } \\
\text { effectiveness }\end{array}$} & \multirow[b]{2}{*}{ Cost* } & \multirow[b]{2}{*}{$\begin{array}{l}\text { Incremental } \\
\text { cost* }\end{array}$} & \multirow[b]{2}{*}{ ICER } \\
\hline & & Baseline & 12-months & & & & & \\
\hline Ohip-Edent & $\begin{array}{l}C D \\
\text { OVD }\end{array}$ & $\begin{array}{l}9.1 \\
11.7\end{array}$ & $\begin{array}{c}10.5 \\
5.7\end{array}$ & $\begin{array}{l}1.4 \\
-6.0\end{array}$ & -7.4 & $\begin{array}{l}354.88 \\
642.96\end{array}$ & 288.08 & (38.93) \\
\hline $\begin{array}{l}\text { Satisfaction } \\
\text { (mandible) }\end{array}$ & $\begin{array}{l}\text { CD } \\
\text { OVD }\end{array}$ & $\begin{array}{l}65.1 \\
51.8\end{array}$ & $\begin{array}{l}62.9 \\
85.4\end{array}$ & $\begin{array}{l}-2.2 \\
33.6\end{array}$ & 35.8 & $\begin{array}{l}354.88 \\
642.96\end{array}$ & 288.08 & 8.05 \\
\hline
\end{tabular}

* PPP converted values

** Cost for I-point reduction in OHIP-EDENT scores and I-point increase in satisfaction with the dentures

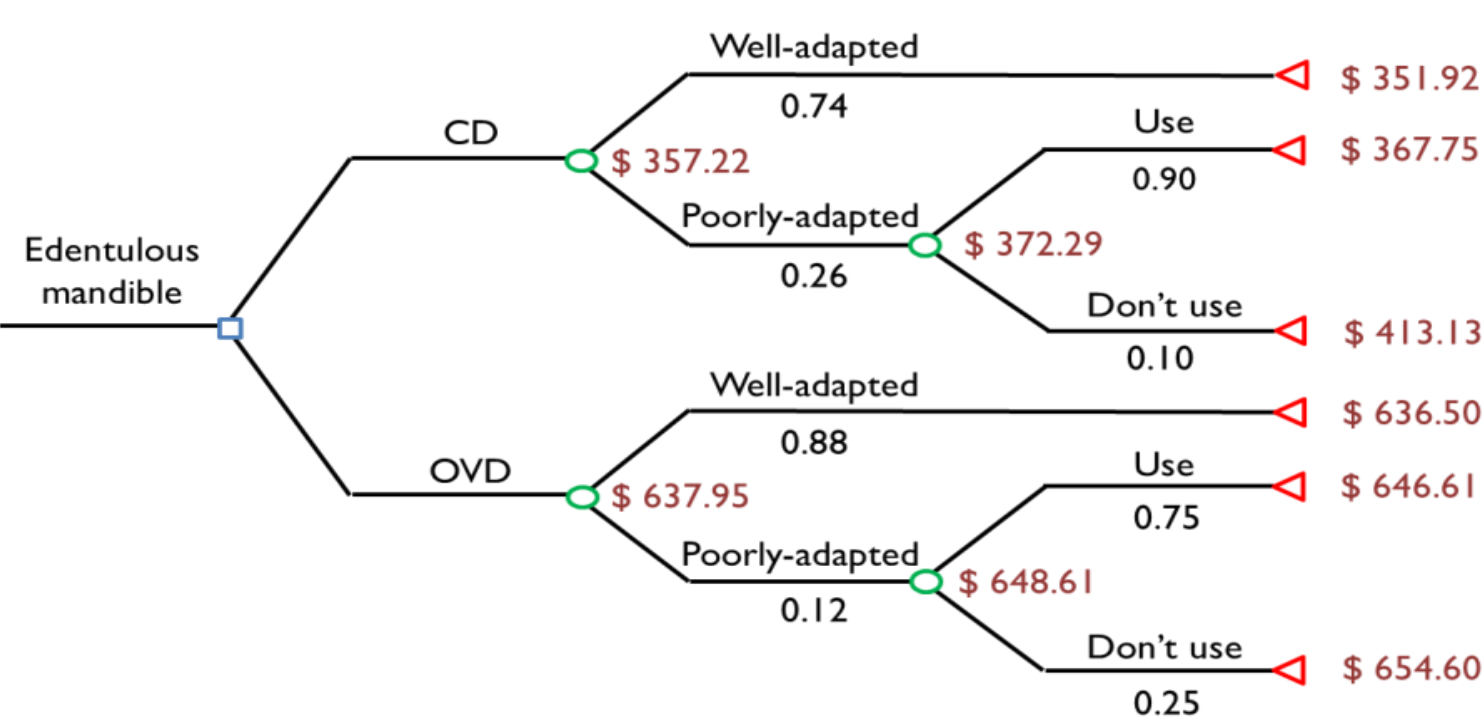

\section{Gonelusions}

- The single implant OVD is a reliable alternative for the edentulous mandible, with significant effect in patient-reported outcomes.

- The relatively low incremental cost (78\%) may potentially increase the utilization of dental implants among older subjects, especially those with limited financial resources.

- Few patients remained unsatisfied with treatment, and a careful selection of patients, based on their expectations and predisposing factors, is essential to choose the best treatment alternative.

Financial support 